\title{
LAS POLÍTICAS DE DESREGULACIÓN DEL TRANSPORTE EN EL ORDEN NACIONAL Y SUBNACIONAL: ASPECTOS DE EFICIENCIA Y EQUIDAD
}

\author{
Tesista: \\ Ana Isabel Talvard \\ Director: \\ Dr. Jorge Barraguirre (h)
}

\section{IDENTIFICACIÓN DE}

La presente investigación se propone describir algunos aspectos institucionales de desregulación de los servicios públicos, fundamentalmente aplicados al transporte. La situación planteada refleja un proceso de reformas en el Estado a partir de los años 1989 que tuvo singular influencia en las distintas áreas del transporte. El transporte sufrió los embates del intervencionismo, siendo objeto de regulaciones que lejos de respetar la transitoriedad para la que en muchas ocasiones se había previsto, se prolongó en el tiempo y favoreció la deformación del Estado dando lugar al llamado "estatismo", bajo cuyo amparo nacieron el déficit fiscal, el endeudamiento interno y externo y la malformación de la Administración Pública. Así fue como a fines de la década de los años 80 la crisis se transformó en terminal y nació una nueva etapa que algunos autores dieron en denominar la "reversión del estatismo"
La investigación se ubica en el período comprendido en el amplio proceso de reformas, a saber: $1^{\circ}$ fase de la reforma que comprende la reducción del aparato estatal, privatizaciones, descentralización tercerización y desregulación de actividades económicas (1989-1994) y la $2^{\mathrm{a}}$ fase -desde 1994 al 2000- fase que queda marcada por la reforma de la Constitución y que marcó su notable influencia en el transporte, área en la que se consolidan los cauces iniciados en 1989 y marcaron proyecciones a futuro.

En este contexto se ubica la política pública de desregulación del transporte, dentro de los cambios producidos con motivo de la Reforma del Estado y en la segunda fase se propone adecuar la distribución de los roles entre las distintas áreas de administración, con criterios de eficacia, eficiencia y economía de gestión.

En cuanto al marco espacial el análisis se circunscribe a los modos más afectados por 
las reformas y que influyeron en la motivación de esta investigación, dado que sufrieron modificaciones distintas. Los modos elegidos para el estudio lo constituyeron el transporte de pasajeros y el transporte de cargas, ambos terrestres. A su vez, en estos modos se estudiaron como influyeron las distintas fases de reformas del Estado en el orden nacional y subnacional, esto es, más precisamente en la Provincia de Santa Fe.

La situación problemática estuvo determinada por la disparidad de procesos y reformas que prima facie, se vislumbraba en los dos modos elegidos para la investigación y en los dos ámbitos: nacional y subnacional.

El enfoque adoptado a esta investigación es el de análisis de las políticas que confluyeron en la desregulación, cómo se desarrollaron los procesos, cómo se distribuyeron las tareas relacionadas con los Organismos nacionales y provinciales, a quienes se les asignaron el rol de Autoridad de Aplicación, que resultados se obtuvieron de ese proceso. En realidad se pretende analizar si la política diseñada tuvo aspectos eficientes y equitativos en el sector en estudio correlativamente con los resultados obtenidos en la práctica.

Se pretende conocer la brecha entre lo diseñado como política de transformación del Estado, si se vio reflejada en el transporte y lo verdaderamente instrumentado. Si la normativa aplicable al transporte en los modos de autotransporte de pasajeros y de cargas elegidos es eficiente para la implementación de los servicios. Analizar si los controles que se imponen en todo el Estado son suficientes, eficientes, garantizan el trato equitativo de los usuarios, o son meramente formales. Si la registración producto de la desregulación del sector es efectiva, coincide con la realidad, con lo producido por dependencias similares de otros ámbitos, o podrían interrelacionarse.

En particular, me interesó toda la problemática planteada tendiente a mejorar el sector, tanto para convertir en efectivos los principios vinculados con la desregulación como para permitir un mejor desempeño del mismo en beneficio ora del Estado, ora de los usuarios y tendientes a su mejoramiento efectivo.

\section{MARCO TEÓRICO}

La problemática descripta-desde el punto de vista teórico- tiene distintas aristas a considerar. Partiendo de la teoría de Oszlack y O'Donnell sobre el análisis de políticas públicas, considerando como tal una toma de posición del Estado frente a determinada "cuestión" y las consideraciones acerca del Estado y de sus relaciones con la Sociedad y el Mercado.

Así y para fundamentar el avance de la investigación se analizó la crisis del Estado, el Consenso de Washington y la crisis fiscal.

Recurrrí a plantear la regulación como política pública, la regulación desde la economía, explicar los monopolios naturales y la regulación desde lo jurídico, para incursionar luego en las distintas teorías sobre regulación, a saber: la clásica del interés público, la del acuerdo colectivo, dentro de la teoría económica de las regulaciones: la escuela de la decisión pública, la teoría de la burocracia y la de los derechos de propiedad.

Todo el proceso de reforma del Estado va a tener una gran influencia en los "servicios públicos", toda vez que las políticas de Estado, de mercado y la tendencia desregulatoria y desmonopolización de actividades conllevarán las nuevas ideologías de los servicios públicos donde aparecen nuevos marcos regulatorios, se crean entes reguladores, entre otras cuestiones.

Teniendo en cuenta el carácter de servicios públicos que adquiere el transporte, exige una primera toma de posición con relación al tema. Implica asumir que persiste la responsabilidad estatal por la garantía de regularidad, uniformidad y continuidad de los 
servicios, más allá de la persona jurídica que esté a cargo de la gestión empresaria. Supone entonces, que la garantía de un acceso equitativo a las redes de servicios, la armonización entre los diferentes operadores y el control de los precios y las normas técnicas de calidad deben ser objeto de reglamentación.

En este punto, desarrollé las distintas teorías por las que atravesara el concepto de "servicio público", desde la "publicatio" del derecho español que significa el traspaso al Estado de la titularidad del senvicio, o la del "public utility" del modelo norteamericano, sistema de prestación privada y la influencia que produjera en el mismo el proceso de privatización.

En resumen, la supervivencia del servicio público como institución jurídica, se hace patente en la Argentina a raíz del proceso de privatizaciones impulsado desde la sanción de la LRE en el año 1989 y confirmada a la luz de las declaraciones legislativas posteriores, incorporadas a los marcos regulatorios de cada sector (art. 42 Constitución Nacional) que prescribe el deber de las autoridades de proveer a la calidad y eficiencia de los servicios públicos.

\section{OBJETIVOS ABORDADOS}

Objetivo General: Analizar las políticas públicas en las cuales se enmarcaron los procesos de desregulación del transporte.

Objetivos Particulares:

1) Identificar cómo se desarrolló dicho proceso de desregulación del transporte en el ámbito nacional.

2) Enmarcar la política de desregulación en el ámbito de la Provincia de Santa Fe.

3) Analizar cómo influyó la desregulación en los dos modos distintos del transporte, como el automotor de pasajeros y de cargas.

4) Determinar los aspectos de eficiencia $y$ equidad surgidos del proceso de desregulación

5) Proponer algún diseño de fortalecimiento del sector en estudio.
4. HIPOTESIS DE INVESTIGACION

1) En el sector del transporte no se han contemplado fehacientemente las transformaciones propuestas en relación a los principios de transformación del Estado.

2) Los aspectos de eficiencia y equidad declamados en oportunidad de sancionarse la Ley de Reforma del Estado no se han cumplido, como así tampoco en la regulación de los servicios públicos.

3) De acuerdo a los roles asignados a cada sector del transporte una vez producida la desregulación se demostró un importante déficit de la capacidad institucional para cumplir su misión en mérito a resguardar el interés público y el de los usuarios.

\section{METODOLOGÍA}

El tipo de investigación se realizará básicamente con aspectos descriptivos y comparativos. El mismo comprende aspectos descriptivos, y básicamente cualitativos a efectos de permitir un análisis comparativo, que sólo se asocia con información cuantitativa para permitir algún tipo de determinación de relaciones o incidencias. El enfoque adoptado a esta investigación es el de análisis de las políticas que confluyeron en la desregulación, cómo se desarrollaron los procesos, cómo se distribuyeron las tareas relacionadas con los Organismos Nacionales y provinciales, a quienes les asignaron el rol de Autoridad de Aplicación, que resultados se obtuvieron de ese proceso. En realidad se pretende analizar si la política diseñada tuvo aspectos eficientes y equitativos en el sector en estudio correlativamente con los resultados obtenidos en la práctica. Se realiza un relevamiento de toda la legislación aplicable en la materia entendiendo por tal su terminología en sentido amplio, es decir, leyes, decretos, normas genéricas dictadas por el P.E. que motivaron las transformaciones del sector del transporte. Se compararon las 
legislaciones en ambos niveles -nacional y subnacional- y se utilizaron como variables eficiencia y equidad. Para ello, liminarmente se definieron las variables elegidas, dado que las mismas resultan de suma importancia para la argumentación de esta investigación, ya que se pretende demostrar si la desregulación del transporte alcanza aspectos de eficiencia y equidad a través de las Políticas Públicas instaura en los ámbitos descriptos. Asimismo, para complementar la definición se eligieron sus dimensiones, a saber: dimensión económica, jurídica y política respecto a la variable eficiencia y dimensión social y política respecto a la variable equidad.

- Eficiencia: es la relación existente entre el trabajo desarrollado, el tiempo invertido, la inversión realizada en hacer algo y el resultado logrado.

- Dimensión Económica: en términos económicos -en el sentido de Pareto- es cuando no es posible mejorar el bienestar de ninguna persona sin empeorar el de alguna otra. Así la competencia perfecta genera una asignación eficiente de los recursos en el sentido de que no hay despilfarro de recursos.

- Eficiencia productiva: consiste en la minimización de costos de producción.

- Eficiencia Asignativa: consistente en que los precios de los bienes y factores reflejen su verdadera escasez. También se hace referencia a la eficiencia distributiva en cuanto a que el excedente social es retenido por los consumidores, quienes supuestamente tienen un mayor peso que los dueños de la empresa dentro de la función de bienestar social.

Respecto a la regulación se considera como una cuestión política, si no se contempla el aspecto de la ciudadanía no se concibe a las instituciones como formas de relación que ayuden a definir el modo político de vida de una sociedad. Sólo la interacción efectiva entre los organismos reguladores y los ciudada- nos puede asegurar su legitimidad y permitirá dar prueba de una modalidad diferente en el ejercicio del poder.

- Dimensión Jurídica: la doctrina manifiesta que un marco jurídico es eficiente cuando logra no sólo una efectiva vigencia normativa, sino que además consigue concretar en la realidad los fines buscados por el legislador al tiempo de sancionarse la norma.

Dicha norma será eficiente si la legislación logra en la práctica que mediante o como corolario de la privatización alcancen ciertos objetivos: 1) del modo establecido; 2) en el tiempo previsto y 3 ) con los efectos buscados.

- Dimensión Política: Encuentra raigambre constitucional en el art. 42 de la $\mathrm{CN}$ al decir:

“Las autoridades proveerán a la protección entre otros... a la defensa de la competencia contra toda forma de distorsión de los mercados, el control de los monopolios naturales y legales, al de la calidad y eficiencia de los servicios públicos..."

También establece que

“...la legislación establecerá procedimientos eficaces para la solución de conflictos y los marcos regulatorios de los servicios públicos de competencia nacional, previendo la necesaria participación de las asociaciones de consumidores y usuarios $y$ de las provincias interesadas, en los organismos de control".

- Equidad: Justicia distributiva basada en la igualdad y proporcionalidad. Moderación en la aplicación de la ley, atemperando según el criterio de justicia el rigor de la letra.

- Dimensión Social: vinculada con las capacidades regulatorias que construye la sociedad 
civil como defensa ante expropiaciones de su poder de decisión por parte de poderes monopólicos.

- Dimensión Política: la misma norma del art. 42 de la CN consagra el principio, al decir:

“...los consumidores y usuarios de bienes y servicios públicos tienen derecho (...) a condiciones e trato equitativo y digno".

En mérito a las pautas que diseñara Guido Santiago Tawil en su artículo "Servicio Público: Eficacia o desgobierno?" dice el autor:

“...Los principales problemas surgidos en la transformación estatal parecen girar alrededor de las formas utilizadas -falta de transparencia, maraña normativa, modificación de pautas previamente establecidas, ausencia de un sistema eficaz de control, protección insuficiente para los usuarios y demás desprolijidades" -que de los fines perseguidos, sea que ellos respondan a concepciones ideológicas determinadas o constituyan una respuesta esencialmente pragmática - tal como podría calificarse a la esbozada por el gobierno argentino - producto de fenómenos típicamente coyunturales-. En esas condiciones, los problemas surgidos en algunos casos en materia de tarifas, atención al público, etc. parecen encontrarse necesariamente relacionados a una deficiente o insuficiente planificación estatal o eventualmente, a una escasamente efectiva actividad de fiscalización".

Adoptando las pautas mencionadas, basé la investigación tomando como indicadores de análisis:

a) Marcos Regulatorios.

b) Sistema tarifario.

c) Atención al público.
Ellos en relación a los distintos ámbitos nacional y subnacional y a los dos modos fijados en los objetivos, esto es, autotransporte de pasajeros y de cargas; en mérito a aceptar la teoría expuesta precedentemente por el autor mencionado y que acepté aplicar a esta investigación, por entender que resulta el marco más descriptivo de la problemática global, aspectos que me permiten emplearlos para fundamentar el trabajo, más precisamente desde la órbita del Estado y su rol en este sector.

\section{CONLUSIONES}

En mérito a las hipótesis de trabajo diseñadas en el punto 3 y como resultado de la investigación desarrollada se pueden aportar las siguientes conclusiones:

1) En el sector del transporte no se han contemplado fehacientemente las transformaciones propuestas en relación con los principios de transformación del Estado.

1.1) En el ámbito nacional es donde más se ha reflejado la reforma, considerándola desde las modificaciones sufridas en los marcos regulatorios.

La desregulación consagrada por el régjmen de apertura económica no alcanzaba al transporte automotor de pasajeros de larga distancia. Con sustento en el proceso de desregulación de la economía argentina enunciada en el Decreto 2284/92, generando un marco normativo de flexibilización en lo concerniente a la prestación de servicios que integran el sistema de transporte. Sin embargo, quedó demostrado -por la opinión de los autores mencionados- en el caso del transporte de pasajeros no operó una desregulación total, sino una apertura de la oferta, con una mayor flexibilización de los servicios. Es lo que los autores dieron en llamar una desregulación dentro de la regulación, dado que deja intacto el sistema que le da sustento al régimen de transporte con son los servicios 
público o también llamados líneas regulares, pero paralelamente se generan servicios de tráfico libre y se da mayor flexibilización a los bloques de turismo y ejecutivos

Hubo una mayor oferta de servicios, se mantuvo el nivel de prestación sin bajar la calidad y descendieron los valores tarifarios -desde el punto de vista del usuario.

1.2) En el transporte de cargas se liberó y desreguló el transporte, como también la carga y la descarga de mercaderías y la contratación entre los transportistas y los dadores de cargas en el territorio nacional. En orden a las transformaciones impuestas por el Poder Ejecutivo se dictó el Decreto $N^{\circ}$ 1494 como marco regulatorio en la materia. Pero recién coincidiendo con la segunda fase de reformas cuando se sancionó la Ley de Cargas No 24653 (1996), la que se aplica a todo traslado de bienes de un lugar a otro en un vehículo por la vía pública, con un fin económico directo y a las actividades conexas al servicio de transporte. Esta ley deja sin efecto no normado anteriormente por las leyes vigentes.

1.3) En el ámbito provincial estas transformaciones no fueron correspondidas, pese a que por la Ley Provincial № 10787 la provincia adhiriera a los principios desregulatorios del Decreto Nacional No 2284/91. La Provincia no compartió el criterio nacional en cuanto a promover la desregulación del transporte automotor de pasajeros implementada en aquella jurisdicción.

Por imperio del artículo $2^{\circ}$ de la Ley 10.787 el P.E. dispuso, en la esfera de su competencia que se adecuaran los Decretos, Resoluciones y disposiciones emanadas de él que contraríen los principios de desregulación a los cuales se adhiere.

Entendiendo que el autotransporte de pasajeros provincial ha logrado una considerable expansión, se encuentra regido por normativas de más de cincuenta años que si bien cons- tituyen un verdadero "código de transporte", hay que adecuarlas a los tiempos modernos para hacer posible la implementación de servicios de autotransporte eficientes y bajo costo para los usuarios.

En el transporte de cargas, si bien el régimen es libre en cuanto a la contratación y fijación de tarifas, se dictó una normativa de menor jerarquía -Decreto № 1041/92- siguiendo los principios de desregulación de la economía, en función de los cuales el transporte de cargas por carretera dejó de ser considerado un servicio público.

No obstante, el desafío consiste en avanzar en el funcionamiento de un marco institucional-regulatorio, y para ello se requiere un marco jurídico político preciso que evite instalar diferencias de mercado, Ello, implica un cambio de modalidad en la aplicación de las reglas de mercado en donde deberá prevalecer la defensa del bien común.

2) Los aspectos de eficiencia y equidad declamados en oportunidad de sancionarse la Ley de Reforma del Estado no se han cumplido, como así tampoco en la regulación de los servicios públicos.

2.1) En el ámbito nacional en el marco de la Reforma del Estado surge un nuevo marco regulatorio, que en realidad significó una desregulación dentro de un mismo sistema, o sea, a todos los que estaban dentro de un sistema se le daba el acceso a todo el Sistema Nacional. Vale decir, que por la vía del tráfico libre podían operar en distintos recorridos interjurisdiccionales. La idea del P.E. era contemplar, ante la hipótesis de una mayor demanda, que las empresas permisionarias podían necesitar un parque móvil mayor durante determinadas etapas de año. Así se permitió el arriendo. Ello trajo consecuencias nefastas, dado que algunos buscaron la forma de entrar al sistema por el costado y mediante un prestador ya instalado y con permiso, otro 
fuera del mismo, acordaba la figura de la locación para ocupar determinado espacio.

A la fecha de esta investigación no se obtuvieron índices de que este cambio resultara "eficiente". Desde el punto de vista del usuario hubo una mayor oferta de servicios, se mantuvo el nivel de prestación sin bajar la calidad y descendieron los valores tarifarios. Para los servicios libres la tarifa también era libre, i obviamente era muy difícil que dentro de ese juego de oferta y demanda, sus tarifas superaran el valor oficial. En algunos recorridos hubo una mayor oferta de servicios. En relación con el usuario y en concordancia con la norma del Art. 42 de la $\mathrm{CN}$ y en pos de velar por la defensa de los intereses del consumidor, la Gerencia de Calidad y Prestación de servicios se conformó con la finalidad de proteger los derechos de los usuarios a través de la comunicación con la comunidad y resolución de sus reclamos y la recepción de sugerencias. Pero, ellos no lograron la representación en esa estructura.

Por imperio de la desregulación en el sector, se produjo una baja significativa de las tarifas, a la vez que se diversificó considerablemente la oferta; y además, la desregulación produjo algunos efectos no queridos que en alguna manera la desnaturalizaron.

Pero, a fin de corregir los efectos no deseados de la desregulación, y en aras de los principios declamados de eficiencia y equidad deberán reforzarse los controles; o bien lograr la desregulación total del sector de forma tal que sea el mercado quien determine la cantidad de empresas y los tipos de servicios que servirán una traza determinada.

Respecto a la cuestión tarifaria pone énfasis en la forma de remuneración de la actividad, pues ella involucra la definición de la estructura de costos, la compensación empresaria y de la tarifa, aspectos claves para la observación del modo en que la reglamentación aporta a la eficiencia de las empresas prestadoras. Conforme a los preceptos teóricos la eficiencia estaría dada por la economía de recursos en la producción de resultados. El objeto de haber colocado valores tarifarios máximos encuentra su razón de ser en alcanzar que a través de la competencia los precios por los viajes tiendan a descender.

En el año 2001 se inicia el camino tendiente a otorgar subsidios al transporte de pasajeros y cargas mediante la utilización de una tasa sobre el expendio del gas oil y el cómputo del pago de los combustibles, para cumplir el pago de otro tipo de impuestos. También se agrega la compensación tarifaria a ciertos operadores de servicios de transporte automotor, mediante el mecanismo del subsidio dentro de la operación.

En el modo del transporte de cargas no se pueden evaluar a la fecha de esta investigación el grado de eficiencia de la normativa sancionada -Ley 24653- toda vez que los aspectos más destacados de la misma y que marcan la diferencia están íntimamente relacionados con la registración a través del R.U.T.A., que está en vías de implementarse.

2.2) En el ámbito subnacional no se vislumbran destacados aspectos de eficiencia y equidad, toda vez que desde el aspecto reglamentario no se cuenta con un plexo normativo acorde con las circunstancias vividas.

Ello, en virtud que el Organismo que los rige fue dictando normas complementarias -creando servicios especiales- con normas de menor rango jurídico, situación que generó discusiones jurídicas por parte de quienes entendían que todos los nuevos servicios no enmarcados en le Ley de Transporte originaria 2499 debían crearse por ley. Situación que a la fecha no está resulta, pese a haberse trabajado en diversos proyectos de nueva ley de transporte provincial que contemple todos los nuevos servicios impuestos por el mercado en la actualidad, y por ende, imposible de desterrar, sin provocar una grave crisis social. 
No obstante, dichos proyectos no han conseguido el consenso parlamentario para permitir su promulgación, ya que los mismos son cooptados por los principales de grupos de poder que sienten que la nueva legislación le ocasionaría un perjuicio, o bien aggiornarse a los tiempos modernos, quizás con gran escasez de recursos para ello.

Desde esta óptica, el Estado deberá agudizar los controles exhaustivamente en cada rubro, para permitir la coexistencia de todos en el marco de una regulación moderada, toda vez que, la desregulación tal como la planteara la Nación no encuentra suficiente madurez empresarial ni facultades exiguas de control por parte del Estado Provincial, como para instrumentarlo en este ámbito subnacional.

En materia tarifaria es muy difícil encontrar en los parámetros implementados en el ámbito subnacional los principios que rigen en el sistema en virtud que correspondería conseguir una prestación eficiente del servicio y no en el establecimiento de tarifas "políticas", que enervan la inversión y la expansión de las instalaciones y bienes necesarios para el servicio. Pese a haberse trabajado en reiterados estudios de costos, falta lograr la suficiente imparcialidad que la situación reclama para permitir la aplicación de los postulados básicos haciendo de las tarifas un moderado, razonable y justo retorno de las inversiones y haciendo posible la aplicación de los principios constitucionales de trato equitativo y digno, no concretados en la práctica.

En cuanto a la atención del usuario, si bien ha comenzado a transitarse el pasaje al paradigma de la Administración Pública orientada al ciudadano, considerado como "cliente" o "usuario", estamos lejos de poder obtener indicadores de eficiencia y equidad en los aspectos propuestos para la investigación.

Esta descripción también es válida para el modo de transporte de cargas en el orden subnacional, donde las tareas de registración y fiscalización están lejos de brindar aspectos de eficiencia y equidad, también por verse en la necesidad de reforzar el sector con recursos humanos y capacitación especializada a las tareas que necesita el área. A más, de una toma de decisiones que conlleve un fuerte compromiso de parte de las autoridades políticas que coadyuve a un diseño y planificación de políticas para el sector.

3) Los objetivos y competencias asignadas a los organismos reguladores están ceñidas a concretar una eficiente administración de sus potestades, dado que se le han otorgado los instrumentos y medios para concretar el bien común.

No obstante, este es el déficit de capacidad institucional más pronunciado, toda vez que las normas regulatorias aplicables al transporte sufren en la práctica resultados distintos a aquellos para los cuales fueron diseñados.

Es importante referenciar las funciones de contralor, fiscalización y aplicación de regímenes sancionatorios que no son todo lo exigente y estrictos que el sistema requiere, para permitir obtener de él los aspectos de eficiencia y equidad que las normas constitucionales le impusieron en pos del bien común.

La falta de procedimientos transparentes en los aspectos de fiscalización y sancionatorios no ayudan a asegurar un proceso de evaluación equitativo y aceptable.

Otro importante déficit se encuentra en la imposibilidad de concretar disposiciones constitucionales en base a proyectos del sector que podrían no cuestionarse pero que en la práctica, tropiezan con serios obstáculos para su concreción. Esto es válido para los reiterados proyectos legislativos nunca concretados en el orden subnacional.

Otro importante déficit de capacidad institucional refiere a problemas presupuestarios que conllevan escasez de personal especiali- 
zado suficiente para cubrir las funciones más claves como las de control y fiscalización y de otros puestos de trabajo para mejorar la atención al público.

Quizás esta investigación pueda senvir de disparador para futuras investigaciones tendientes a diseñar un programa de fortalecimiento institucional que permita convertir al sector en esferas verdaderamente eficientes y equitativas para satisfacer el bien común tal como fueran los propósitos de la desregulación.

\section{BIBLIOGRAFÍA}

Bustamante, Jorge Eduardo (1993): Desregulacion entre el Derecho y la Economía. Buenos Aires, Ed. Abeledo Perrot.

Canosa, Armando (2002): El Derecho Administrativo del Transporte Terrestre. Buenos Aires, Ed. Abaco.

Mairal, Héctor: "La ideología del Servicio Público" en Revista de Derecho Administrativo No 14, Buenos Aires, Depalma.

Mata, Ismael (2000): Una visión sesgada de la regulación en Revista del Régimen de la Administración Pública. Año XXII, № 256, pp. 101-107.

Nallar, Daniel M. (1999): El Estado Regulador y el Nuevo Mercado del jurídico Servicio Público. Análisis sobre la privatización, la regulación y los entes regulatorios Prólogo de Cassagne, Buenos Aires, Depalma.

Olivieri, Roberto Luis (1996): Transporte por automotor de pasajeros en el ámbito interurbano e internacional, Buenos Aires.

Oszlack, Oscar y Ruth Felder: La capacidad de regulación estatal en la Argentina. Quit custodiet Custodes? en "La Argentina que viene. Análisis y propuestas para una sociedad en transición. ISUANI, Aldo-FILMUS, Daniel Grupo Editorial, NORMA-FLACSO.

Tawil, Guido Santiago (1991): Servicios Públicos: eficacia o desgobierno, La Ley -C- p.639. 